\title{
NUMERICAL INVESTIGATION OF THE MAIN CHARACTERISTICS OF HEAT AND MASS TRANSFER WHILE HEATING THE HETEROGENEOUS WATER DROPLET IN THE HOT GASES
}

\author{
Maxim V. Piskunov ${ }^{1,}$, , Anastasia A. Shcherbinina ${ }^{1}$, Olga V. Vysokomornaya ${ }^{1}$ \\ ${ }^{1}$ National Research Tomsk Polytechnic University, 634050 Tomsk, Russia
}

\begin{abstract}
The processes of heat and evaporation of heterogeneous water droplet with solid (by the example of carbon) inclusion in hot (from $800 \mathrm{~K}$ to $1500 \mathrm{~K}$ ) gases were investigated by the developed models of heat and mass transfer. We defined the limited conditions, characteristics of the droplet and the gas medium which are sufficient for implementing the "explosive" destruction of heterogeneous droplet due to intensive vaporization on an inner interface, and intensive evaporation of liquid from an external (free) droplet surface. The values of the main characteristic of the process (period from start of heating to "explosive" destruction) obtained in response to using various heat and mass transfer models were compared.
\end{abstract}

\section{Introduction}

The thematic justification of phase transformation of droplets and water droplet flows as well as solutes, emulsions and suspensions on its base while moving in hot gases is due to a large number of practical applications of the results (polydisperse fire extinguishing; thermal purification of water, emulsions and suspensions on its base; cleaning and processing of contaminated technological surfaces and others) [1,2]. Except droplet disintegration to typical sizes of several micrometers, using the heterogeneous droplets consisting of solid (e.g. carbon) inclusion and liquid fuel enveloping it can be one method of increasing the intensity of heat and mass transfer during injection droplet flows into the region of hot gases. In this case, the solid particles can accumulate the energy supplied to its surface through the film of water by means of convective, conductive and radiative heat transfer. The particle heated in such way (inclusion) within the droplet becomes an additional source of heat, initiating a phase transformation at the inner interface.

The purpose of this work is to conduct the numerical investigations of heat and mass transfer and phase transformations while heating the heterogeneous water droplet with solid inclusion in hot gases.

\section{Problem statement and solution methods}

Fig. 1 presents the scheme of the region of numerical solution of heat transfer problem.

${ }^{a}$ Corresponding author: piskunovmv@tpu.ru 


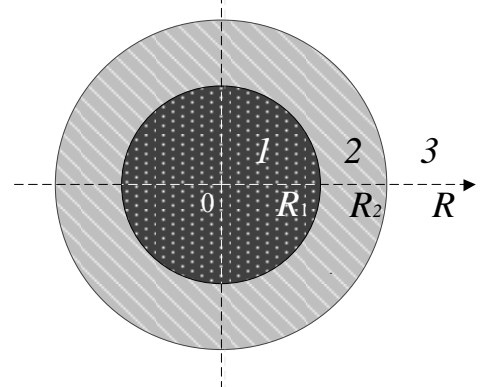

Figure 1. Scheme of the numerical solution domain of heat transfer at $0<t<t_{\mathrm{h}}$ : $1-$ inclusion, $2-$ liquid, $3-$ hot gases.

Water droplet (external radius $R_{2}$, dimension of solid inclusion $R_{1}$ ) with initial temperature $T_{0}$ is in medium of hot gases with temperature $T_{\mathrm{f}}$. There is a heat-up of heterogeneous droplet and liquid evaporation from an external (free) surface. A part of the emission energy of the hot gas medium due to radiative water diathermancy becomes to inert interface "inclusion - liquid" $\left(R=R_{1}\right)$. If reaching the temperature corresponding to intensive phase transformations (e.g. boiling - for water $370 \mathrm{~K}$ ) at an inert interface of heterogeneous droplet, the formation process of vapor layer is accelerated in the vicinity of the contact line of liquid film with solid particle. On further heating of heterogeneous droplet, pressure and temperature of the vapor on the internal interface grows, that can lead to an "explosive" disintegration of liquid film with forming smaller droplets. When the temperature at inert interface $R=R_{1}$ reaches the value of 370 , it is considered as necessary and sufficient condition for "explosive" vaporization while heating of a heterogeneous droplet in the system (Fig. 1).

In conducting the investigations we developed several models that take into account various effects and allow analyzing the influence on heat and mass transfer intensity of a large group of factors (the main mathematical models are presented in the works [3-5]).

Nonlinear nonstationary differential partial equations describing the physical formulations [3-5] were solved by finite difference method, the finite-difference analogs of the original differential equations by a locally one-dimensional method and method of variable directions, nonlinear equations by the method of iterations. To solve the one-dimensional difference equations we applied the sweep method using a four-point implicit difference scheme. To assess the reliability of the results of numerical investigations, it was performed the verification of conservative of difference scheme.

\section{Results and Discussion}

On conducting the numerical investigations, the external radius of heterogeneous droplets varied within the range $R_{2}=0.5 \div 1 \mathrm{~mm}$, thickness of water film within $\delta R=0.1 \div 0.5 \mathrm{~mm}$; temperature of gas medium within $T_{\mathrm{f}}=800 \div 1500 \mathrm{~K}$, the initial temperature of heterogeneous droplet within $T_{0}=300 \mathrm{~K}$. The thermophysical and optical properties of water, air, combustion products of liquid fuel and carbon particle were selected according to these [6].

The numerical model developed for studying the heat and mass transfer [3] allows us to analyze the influence on the main characteristic of the process - typical period till the realization of intense evaporation on the inert boundary $t_{\mathrm{h}}$, it means the influence of such parameters of the system as temperature of gas medium $T_{\mathrm{f}}$, initial size of droplet $R_{2}$ and solid inclusion $R_{1}$, thickness of liquid film $\delta R$, its optical properties (in particular, $k_{\lambda}$ ).

The results of applying the model [3] allowed determining the extreme values of gas medium temperature and typical sizes of heterogeneous droplet when the effect of "explosive" disintegration is possible due to intense vaporization at the contact boundary "water film - solid carbon inclusion": $T_{\mathrm{f}} \geq 1050 \mathrm{~K}$ and $R_{2} \geq 0.3 \cdot 10^{-3} \mathrm{~m}$. Also, we defined by the model [3] that there are some extreme values of film $\delta R$ at which the realization of intensive vaporization conditions at the interface inside droplet is possible. As such, we can select $\delta R<0.2 \cdot 10^{-3} \mathrm{~m}$. It was found that at low film thickness, the 
water evaporates intensively from the outer boundary of the droplets for a few seconds in case of failure of temperature $T\left(R_{1}\right) \approx 370 \mathrm{~K}$.

In addition, on conducting numerical investigations, the influence on intensity of heat and mass transfer and phase transformation of radiation absorption coefficient by water $k_{\lambda}$ was established. The results of numerical simulation allow concluding that variation of absorption coefficient within the range $k_{\lambda}=10^{-3} \div 10 \mathrm{~m}^{-1}$ does not practically influence the time $t_{\mathrm{h}}$. With a further gain in the absorption coefficient $k_{\lambda}$, the typical time $t_{h}$ markedly increase. The "threshold" value $k_{\lambda}$ at which growth of parameter $t_{\mathrm{h}}$ becomes noticeable, is $2 \cdot 10^{2} \mathrm{~m}^{-1}$. This result is quite logical, since the value of $k_{\lambda}$ is inversely proportional to the distance, which the optical radiation emitting in the substance can overcome.

With further development of the physical setting of the studying process, the physical and mathematical model [3] was extended to assess the impact of flow gap availability on the inert interface $R=R_{1}$ on the rate of evaporation of the heterogeneous droplet, as well as the supply of energy to the interface through a solid inclusion and film liquid [4]. Phase transformations at the boundary $R=R_{1}$ leads to decrease in characteristic thickness of water film around inclusion. As a consequence, droplet size decreases and thickness of vapor layer growths, that leads to domination of endothermic transformations at inert interfaces in a droplet in comparison with that realized at the free surface.

The Table presents the evaluation results of influence of heat and mass transfer mechanisms in a heterogeneous droplet while heating in high-temperature gases.

Table. Periods of "explosive" vaporization under using different models [3-5].

\begin{tabular}{|c|c|c|c|c|c|c|c|c|}
\hline & \multicolumn{8}{|c|}{ Temperature of gas medium, $\mathrm{K}$} \\
\hline & 800 & 900 & 1000 & 1100 & 1200 & 1300 & 1400 & 1500 \\
\hline$t_{\mathrm{h} 1}, \mathrm{~s}$ & \multicolumn{5}{|c|}{ no vaporization at the boundary $R=R_{1}$} & 7.269 & 1.984 & 0.752 \\
\hline$t_{\mathrm{h} 2}, \mathrm{~s}$ & 8.154 & 4.607 & 2.315 & 1.397 & 0.698 & 0.182 & 0.016 & 0.009 \\
\hline$t_{\mathrm{h} 3}, \mathrm{~s}$ & \multicolumn{4}{|c|}{ no vaporization at the boundary $R=R_{1}$} & 2.901 & 1.253 & 0.715 & 0.603 \\
\hline
\end{tabular}

$t_{\mathrm{h} 1}$ - the results of using the model without taking into account the formation of a vapor layer at the boundary $R=R_{1}$;

$t_{\mathrm{h} 2}$ - the results of using the model taking into account the formation of a vapor layer at the boundary $R=R_{1}$;

$t_{\mathrm{h} 3}$ - the results of using the model taking into account the supply of energy to an interface through a solid inclusion and liquid film.

The values of typical periods $t_{\mathrm{h}}$ presented in the table allow concluding on a high role of thermal and physical characteristics if inclusion material. If heat conduction of inclusion is higher than for liquid, the periods $t_{\mathrm{h}}$ can be decreased with a larger inclusion overriding the droplet surface (most of energy of gases will be supplied to inert interfaces through a particle, rather than liquid). At a lower thermal conductivity of inclusion relative to the liquid, it seems advisable to use a different effect accumulation of energy passing through the liquid on the surface of the nontransparent inclusion with low thermal conductivity.

Physical features selected at simulation can explain also characteristic minimal temperatures of gases at which an "explosive" vaporization is possible for different heat and mass transfer models. In particular, from the data in the Table we can conclude that the conditions of "explosive" vaporization are realized at gas temperatures of about $800 \mathrm{~K}$ for conditions of energy supplying to an inert interface through an inclusion and liquid. For the other two models, minimum temperatures of gases are higher than this value (1200 K and $1300 \mathrm{~K}$, correspondingly).

\section{Conclusions}

Analyzing the results of the numerical investigations of heat and mass transfer and phase transformations in heterogeneous droplets while heating in high-temperature gases by the models developed before [3-5] allowed determining the ranges of extreme values of parameters of droplet and 
gases when the effect of "explosive" droplet disintegration is possible due to intense vaporization at an inert interface. According to the results of numerical simulation, we can make a judgement on a possibility of applying the water with a wide range of values of absorption coefficient $k_{\lambda}$ without purification and clarification to intensify the phase transformation. Also, as a result of conducted investigations, the values of gas medium temperature typical of various schemes of heat supplying and extreme for realization of "explosive" disintegration were determined.

\section{Acknowledgments}

The study was supported by the grant of the President of the Russian Federation (MD-2806.2015.8).

\section{References}

1. R.S.Volkov, G.V.Kuznetsov, P.A. Strizhak, Int. Journ. of Therm. Scien. 88 (2015).

2. M. Renksizbulut, M.C. Yuen, Journ. of Heat Transf. 105 (1983).

3. G.V.Kuznetsov, M.V. Piskunov, P.A. Strizhak, Int. Journ. of Heat and Mass Transf. 92 (2016).

4. M.V. Piskunov, P.A. Strizhak, A.A. Shcherbinina, Therm. Scien. OnLine-First Issue 00 (2015), p. 164.

5. O.V. Vysokomornaya, G.V.Kuznetsov, P.A. Strizhak, Journ. of Mechanics. August (2015).

6. N.B. Vargaftik, L.P. Filipov, A.A. Tarzimanov, E.E. Totskii, Handbook of Thermal Conductivity of Liquids and Gases, CRC Press Inc, Boca Raton, 1994. 